\title{
ANISOTROPIC CONSTITUTIVE MODEL OF STRAIN-INDUCED PHENOMENA IN STAINLESS STEELS AT CRYOGENIC TEMPERATURES
}

\author{
C. Garion, B. Skoczen
}

\begin{abstract}
A majority of the thin-walled components subjected to intensive plastic straining at cryogenic temperatures are made of stainless steels. The examples of such components can be found in the interconnections of particle accelerators, containing the superconducting magnets, where the thermal contraction is absorbed by thin-walled, axisymetric shells called bellows expansion joints. The stainless steels show three main phenomena induced by plastic strains at cryogenic temperatures: serrated (discontinuous) yielding, $\gamma->\alpha^{\prime}$ phase transformation and anisotropic ductile damage. In the present paper, a coupled constitutive model of $\gamma->\alpha^{\prime}$ phase transformation and orthotropic ductile damage is presented. A kinetic law of phase transformation, and a kinetic law of evolution of orthotropic damage are presented. The model is extended to anisotropic plasticity comprising a constant anisotropy (texture effect), which can be classically taken into account by the Hill yield surface, and plastic strain induced anisotropy. For such a model the shape of the yield surface in the stress space varies as a function of the plastic strains. The constitutive model creates a bridge between material science (experiments) and structural analysis. It has been used to predict the response of beam vacuum and cryogenic bellows to monotonic and cyclic loads developed in the interconnections of the Large Hadron Collider at CERN.
\end{abstract}

Accelerator Technology Department, Geneva, Switzerland

\author{
Presented at the 2003 Cryogenic Engineering Conference and International Cryogenic Materials Conference \\ CEC/ICMC 2003 \\ 22-26 September 2003, Anchorage, Alaska
}

Administrative Secretariat

AT Division

CERN

$\mathrm{CH}$ - 1211 Geneva 23
Geneva, Switzerland

29 January 2004 


\title{
ANISOTROPIC CONSTITUTIVE MODEL OF STRAIN-INDUCED PHENOMENA IN STAINLESS STEELS AT CRYOGENIC TEMPERATURES
}

\author{
C. Garion, B. Skoczen \\ CERN, European Organization for Nuclear Research \\ CH-1211 Geneva 23
}

\begin{abstract}
A majority of the thin-walled components subjected to intensive plastic straining at cryogenic temperatures are made of stainless steels. The examples of such components can be found in the interconnections of particle accelerators, containing the superconducting magnets, where the thermal contraction is absorbed by thin-walled, axisymetric shells called bellows expansion joints. The stainless steels show three main phenomena induced by plastic strains at cryogenic temperatures: serrated (discontinuous) yielding, $\gamma \rightarrow \alpha$ ' phase transformation and anisotropic ductile damage. In the present paper, a coupled constitutive model of $\gamma \rightarrow \alpha$ ' phase transformation and orthotropic ductile damage is presented. A kinetic law of phase transformation, and a kinetic law of evolution of orthotropic damage are presented. The model is extended to anisotropic plasticity comprising a constant anisotropy (texture effect), which can be classically taken into account by the Hill yield surface, and plastic strain induced anisotropy. For such a model the shape of the yield surface in the stress space varies as a function of the plastic strains. The constitutive model creates a bridge between material science (experiments) and structural analysis. It has been used to predict the response of beam vacuum and cryogenic bellows to monotonic and cyclic loads developed in the interconnections of the Large Hadron Collider at CERN.
\end{abstract}

\section{INTRODUCTION}

Structural materials for cryogenic applications, like stainless steel, are known to preserve their ductility also at very low temperatures, close to absolute zero. Ductile behavior implies evolution of plastic strain fields in components subjected to thermomechanical loads under cryogenic conditions. Three principal phenomena, associated with the evolution of plastic strains, are observed in ductile materials at low temperatures:

- The serrated yielding (discontinuous in terms of $\mathrm{d} \sigma / \mathrm{d} \varepsilon$ ) which occurs typically at $4.2 \mathrm{~K}$ (in liquid helium) and for a plastic strain rate above a given critical value (about $10^{-3} \mathrm{~s}^{-1}$ ) $[1 ; 2]$. This effect is not taken into account in the present paper. 
- The second relevant phenomenon is the plastic strain induced phase transformation. The FCC $(\gamma)$ austenitic phase is transformed into BCC $\left(\alpha^{\prime}\right)$ martensite. Plastic strain induced phase transformation is at the origin of considerable evolution of material properties. According to Olson and Cohen [3], the main mechanism contributing to the onset of martensitic transformation is the intersection of shear bands.

- The third phenomenon consists in the plastic strain driven evolution of ductile damage at cryogenic temperatures. This dissipative and irreversible process leads to the creation of a field of microcracks and microvoids (micro-damage) and to the related "softening" of the material. Since the evolution of damage, observed in thin-walled shells subjected to plastic straining at low temperatures shows the features that can not be explained by the isotropic model, an orthotropic model is adopted. Both the martensite platelets and the microcrack fields develop simultaneously and coexist in the same material volume (Fig. 1a, 1b). In the isotropic version, they are described by the volume fraction of martensite $(\xi)$ and the damage parameter (D), respectively. The anisotropic model is based on the assumption of a two-phase material $(\gamma, \alpha)$, represented by the scalar $\xi$ and anisotropic damage, described by the tensor $\underline{\underline{D}}$. The anisotropy of damage is often related to the material texture, which is also reflected by the anisotropy of plastic straining. Here the Hill model $[4,5]$ of yield surface is particularly useful. Finally, in order to describe correctly the damage coupled with plasticity a model based on plastic strain induced anisotropy has to be applied.

1a)

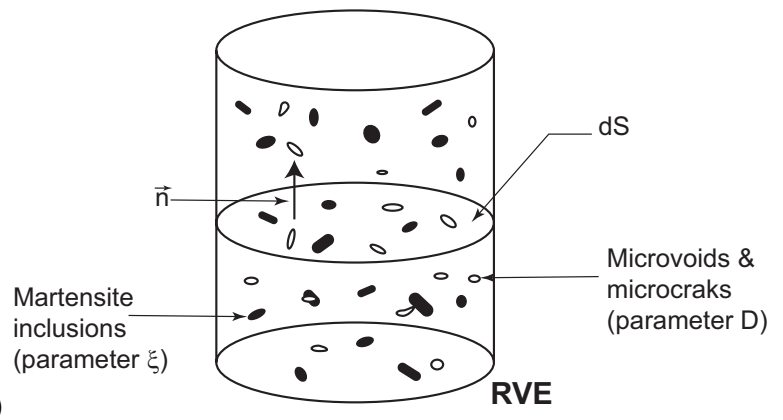

1b)

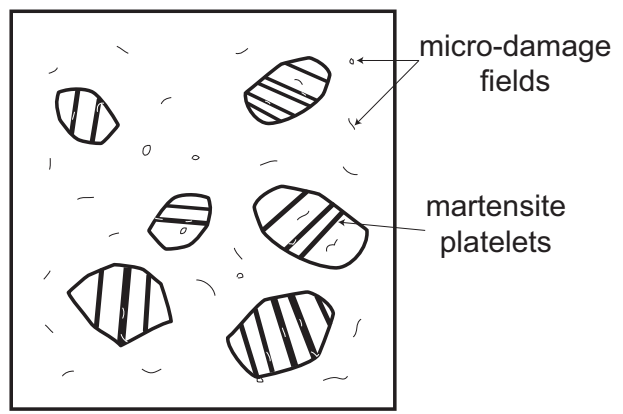

FIGURE 1 The Representative Volume Element (RVE) and a section of damage affected two-phase material

\section{MODELLING OF THE PLASTIC STRAIN INDUCED MARTENSITIC TRANSFORMATION $\left(\gamma \rightarrow \alpha^{\prime}\right)$}

\section{Kinetic law of martensitic transformation}

Kinetics of $\gamma \rightarrow \alpha$ ' phase transformation, as described in [3], is reflected by a typical sigmoidal curve defining the evolution of the martensite content $(\xi)$ as a function of the plastic strain (Fig. 2).

A simplified evolution law for the volume fraction of martensite has been introduced for the linear part (region II) of the sigmoidal curve by Garion and Skoczen [6]:

$$
\dot{\xi}=\mathrm{A}(\mathrm{T}) \dot{\mathrm{p}} \mathrm{H}\left((\mathrm{p}-\mathrm{p} \xi)\left(\xi_{\mathrm{L}}-\xi\right)\right)
$$

where $\dot{\mathrm{p}}$ denotes the accumulated plastic strain rate, defined as:

$$
\dot{\mathrm{p}}=\sqrt{\frac{2}{3} \dot{\dot{\varepsilon}}^{\mathrm{p}}: \dot{\underline{\varepsilon}}^{\mathrm{p}}}
$$

where $\dot{\varepsilon}^{\mathrm{p}}$ is the plastic strain rate tensor. In equation (1), $\mathrm{p}_{\xi}$ denotes the accumulated plastic strain threshold to initiate the phase transformation and $\xi_{\mathrm{L}}$ stands for the saturation level of the martensite content. $\mathrm{H}$ is the Heavyside function. 


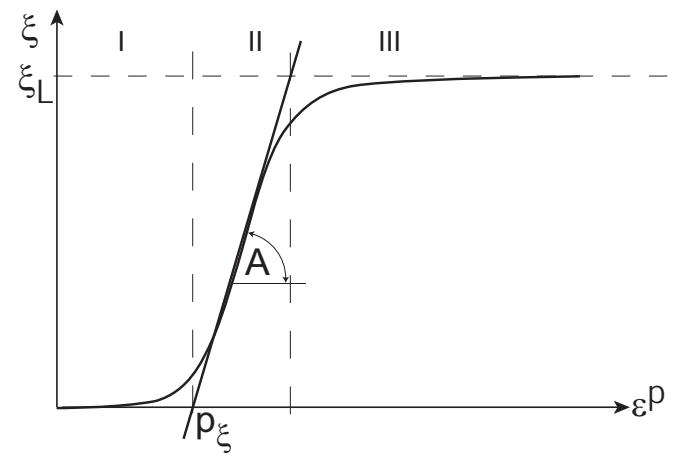

FIGURE 2 Linearization of the sigmoidal curve, martensite content versus plastic strain.

\section{Bi-phase initially isotropic and homogenous material - constitutive model}

The following set of constitutive equations has been developed for a bi-phase $\left(\gamma+\alpha^{\prime}\right)$ isotropic and ductile material [6]:

- The constitutive law:

$$
\underline{\underline{\sigma}}=\underline{\underline{\underline{E}}}:\left(\underline{\underline{\varepsilon}}-\underline{\underline{\varepsilon}}^{\mathrm{p}}-\underline{\underline{\varepsilon}}^{\text {th }}-\xi_{\underline{\underline{\varepsilon}}}^{\mathrm{PT}}\right)
$$

where $\underline{\underline{\varepsilon}}^{\mathrm{PT}}$ denotes the phase transformation strain and $\underline{\underline{\varepsilon}}^{\text {th }}$ stands for the thermal strain. $\underline{\underline{\equiv}}$ is the fourth-rank elasticity tensor.

- The yield surface is defined by:

$$
\mathrm{f}_{\mathrm{c}}(\underline{\underline{\sigma}}, \underline{\underline{\underline{X}}}, \mathrm{R})=\mathrm{J}_{2}(\underline{\underline{\sigma}}-\underline{\underline{X}})-\sigma_{\mathrm{y}}-\mathrm{R}
$$

where $\mathrm{J}_{2}$ is the second invariant of the stress tensor and $\underline{\underline{X}}$ and $\mathrm{R}$ stand for the kinematic and the isotropic hardening variables, respectively. Here $\sigma_{\mathrm{y}}$ denotes the yield point.

- The associated flow rule:

$$
\mathrm{d} \varepsilon^{\mathrm{p}}=\frac{\partial \mathrm{f}_{\mathrm{c}}}{\partial \underline{\underline{\sigma}}} \mathrm{d} \lambda=\frac{3}{2} \frac{\underline{\underline{\mathrm{s}}}-\underline{\underline{X}}}{\mathrm{~J}_{2}(\underline{\underline{\underline{\sigma}}}-\underline{\underline{X}})} \mathrm{d} \lambda
$$

- The hardening law:

$$
\begin{gathered}
\underline{\underline{\dot{X}}}=\frac{2}{3} \mathrm{~g}(\xi) \underline{\underline{\varepsilon}}^{\mathrm{p}} \\
\dot{\mathrm{R}}=\mathrm{f}(\xi) \dot{\mathrm{p}}
\end{gathered}
$$

where the functions $\mathrm{g}(\xi)$ and $\mathrm{f}(\xi)$ are obtained from the Mori-Tanaka homogenization [6]. A good correlation between the model and the experiment is obtained [6].

\section{ORTHOTROPIC DUCTILE DAMAGE}

\section{Second order damage tensor: $\underline{\underline{D}}$}

Assume at a given point a local set of unit base vectors $\vec{n}_{i}$, tangent to the principal directions. The relevant damage tensor is introduced in the form [10]:

$$
\underline{\underline{D}}=\sum_{\mathrm{i}=1,3} \mathrm{D}_{\mathrm{i}} \overrightarrow{\mathrm{n}}_{\mathrm{i}} \otimes \overrightarrow{\mathrm{n}}_{\mathrm{i}}
$$

where $\vec{n}_{i}$ stands for the base vector associated with the principal direction $i$ and $D_{i}$ denotes the component of the damage tensor related to the direction i. It is defined by:

$$
\mathrm{D}_{\mathrm{i}}=\frac{\mathrm{dS} \mathrm{D}_{\overrightarrow{\mathrm{n}}_{\mathrm{i}}}}{\mathrm{dS_{ \vec { \textrm {n } } _ { \mathrm { i } } }}}
$$


where $S_{D_{\vec{n}_{i}}}$ is the area of damage in the section $S_{\vec{n}_{i}}$, represented by the normal $\vec{n}_{i}$.

\section{The effective stress: $\underline{\underline{\widetilde{\sigma}}}$}

The effective stress $\underset{\underline{\sigma}}{\underline{\sigma}}$ is supposed to obey the strain equivalence principle [9]:

$$
\underline{\underline{\widetilde{\sigma}}}=\underline{\underline{\underline{E}}}: \underline{\underline{\varepsilon}}^{\mathrm{e}}
$$

The relation between the stress and the effective stress tensors is postulated under the following form:

$$
\underline{\underline{\sigma}}=\frac{1}{2}((\underline{\underline{I}}-\underline{\underline{D}}) \underline{\underline{\sigma}}+\underline{\underline{\sigma}}(\underline{\underline{I}}-\underline{\underline{D}}))
$$

with $\underline{\underline{I}}$ being the identity tensor. The general relationship between the stress and the effective stress tensors reads:

$$
\underline{\underline{\sigma}}=\underline{\underline{\underline{M}}}^{-1}: \underline{\underline{\sigma}}
$$

Hence, the Helmholtz free energy state potential for linear elasticity coupled with damage is written in the following form:

$$
\Psi=\frac{1}{\rho}\left(\frac{1}{2} \underline{\underline{\varepsilon}}: \underline{\underline{\underline{\underline{M}}}: \underline{\underline{\underline{E}}}: \underline{\underline{\varepsilon}}}\right)+\Psi_{\mathrm{p}}
$$

where $\psi_{\mathrm{p}}$ is the plastic part which does not explicitly depend upon $\underline{\underline{D}}$.

\section{Kinetic laws of damage evolution for orthotropic ductile materials}

In the case of anisotropic ductile damage the damage parameter $\mathrm{D}$ is replaced by the damage tensor $\underline{\underline{D}}$ and the scalar function of the strain energy density release rate $Y$ is replaced by the relevant tensor $\underline{\underline{Y}}$ [9]. Again both $\underline{\underline{D}}$ and $\underline{\underline{Y}}$ form a pair of dual state variables. In the present paper it is assumed that the driving force of evolution of orthotropic ductile damage remains the accumulated plastic strain. Thus, the kinetic law of damage evolution is postulated in the following form [7]:

$$
\underline{\underline{\mathrm{D}}}=\underline{\underline{\mathrm{C}}} \underline{\underline{\mathrm{Y}}} \underline{\underline{\mathrm{C}}}^{\mathrm{T}} \dot{\mathrm{p}} \mathrm{H}\left(\mathrm{p}-\mathrm{p}_{\mathrm{D}}\right)
$$

which assures that the tensor of damage is symmetric. Tensor $\underline{\underline{\mathrm{C}}}$ is defined as follows:

$$
\underline{\underline{C}}=\sum_{i=1,3} \mathrm{C}_{\mathrm{i}} \overrightarrow{\mathrm{n}}_{\mathrm{i}} \otimes \overrightarrow{\mathrm{n}}_{\mathrm{i}}
$$

and can be classified as the symmetric tensor containing the material moduli. The strain energy density release rate tensor is defined by:

$$
\underline{\underline{Y}}=-\rho \frac{\partial \Psi}{\partial \underline{\underline{D}}}
$$

The relevant potential of dissipation reads:

$$
\Phi=\frac{1}{2}\left(\underline{\underline{C}} \underline{\underline{Y}} \underline{\underline{C}}^{\mathrm{T}}\right): \underline{\underline{Y}} \sqrt{\frac{\left(\underline{\underline{\underline{\mathrm{s}}}-\underline{\underline{X}}): \underline{\underline{\underline{M}}}^{-1}: \underline{\underline{\underline{M}}}^{-1}:(\underline{\underline{\underline{\underline{s}}}}-\underline{\underline{X}})}\right.}{(\underline{\underline{\underline{\underline{s}}}-X} \underline{\underline{\underline{X}}}): \underline{\underline{\underline{\mathrm{s}}}}-\underline{\underline{X}})}}
$$

The isotropic conjugate damage variables, $\mathrm{D}$ and $\mathrm{Y}$, can be obtained from the orthotropic state variables $\underline{\underline{D}}$ and $\underline{\underline{Y}}$ by the following operations:

$$
\mathrm{Y}=\operatorname{Tr} \underline{\underline{\mathrm{Y}}}\rfloor
$$

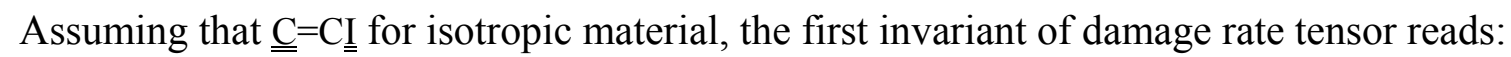

$$
\dot{\mathrm{D}}=\operatorname{Tr}[\underline{\dot{\mathrm{D}}}]=\mathrm{C}^{2} \mathrm{Y} \dot{\mathrm{p}}
$$

which corresponds to the isotropic damage evolution law with $S=1 / C^{2}[8 ; 9]$. 


\section{COMBINED SET OF EQUATIONS FOR A TWO-PHASE MATERIAL WITH ORTHOTROPIC DAMAGE}

The final set of the constitutive equations for a two-phase heterogeneous material with orthotropic ductile damage takes the following form:

- Kinetics of martensitic transformation $\left(\gamma \rightarrow \alpha^{\prime}\right)$ :

$$
\dot{\xi}=\mathrm{A}(\mathrm{T}, \underline{\underline{\sigma}}) \dot{\mathrm{p}} \mathrm{H}\left(\left(\mathrm{p}-\mathrm{p}_{\xi}\right)\left(\xi_{\mathrm{L}}-\xi\right)\right)
$$

- Kinetic law of damage evolution:

- The constitutive law:

$$
\underline{\underline{\mathrm{D}}}=\underline{\underline{\mathrm{C}}} \underline{\underline{\mathrm{Y}}} \underline{\underline{C}}^{\mathrm{T}} \dot{\mathrm{p}} \mathrm{H}\left(\mathrm{p}-\mathrm{p}_{\mathrm{D}}\right)
$$

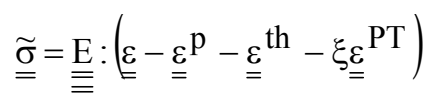

- The stress-effective stress relation:

$$
\underline{\underline{\sigma}}=\frac{1}{2}((\underline{\underline{\mathrm{I}}}-\underline{\underline{\mathrm{D}}}) \underline{\underline{\sigma}}+\underline{\underline{\tilde{\sigma}}}(\underline{\underline{\mathrm{I}}}-\underline{\underline{\mathrm{D}}}))
$$

- Yield surface is defined by:

$$
\mathrm{f}_{\mathrm{c}}(\underline{\underline{\widetilde{\sigma}}}, \underline{\underline{X}}, \mathrm{R})=\mathrm{J}_{2}(\underline{\underline{\widetilde{\sigma}}}-\underline{\underline{X}})-\sigma_{\mathrm{y}}-\mathrm{R}
$$

- The associated flow rule:

$$
\mathrm{d}_{\underline{\varepsilon}}^{\mathrm{p}}=\frac{\partial \mathrm{f}_{\mathrm{c}}}{\partial \underline{\underline{\sigma}}} \mathrm{d} \lambda
$$

- The evolution laws for the hardening parameters:

$$
\begin{gathered}
\underline{\underline{\dot{X}}}=\frac{2}{3} \mathrm{~g}(\xi) \dot{\dot{\varepsilon}}^{\mathrm{p}} \\
\dot{\mathrm{R}}=\mathrm{f}(\xi) \dot{\mathrm{p}}
\end{gathered}
$$

Here, the yield surface has been assumed as being a function of the second invariant of the stress tensor (Huber-Mises-Hencky concept). An extrapolation to the Mroz model [13] is proposed in order to take into account the texture effect. Texture induced anisotropic plasticity is proposed in the following form:

$$
\mathrm{f}_{\mathrm{c}}(\underline{\underline{\widetilde{\sigma}}}, \underline{\underline{X}}, \mathrm{R})=(\underline{\underline{\sigma}}-\underline{\underline{X}}): \underline{\underline{\underline{\underline{A}}}}:(\underline{\underline{\widetilde{\sigma}}}-\underline{\underline{X}})-\left(\sigma_{\mathrm{y}}+\mathrm{R}\right)^{2}
$$

The tensor $\underline{\underline{\equiv}}$ corresponds to the Hill plastic surface, related to the initial texture, or is expressed as a function of the plastic strains. The following simplified formulation is adopted [13]:

$$
\underline{\underline{\underline{\mathrm{A}}}}=\underline{\underline{\underline{\mathrm{K}}}}+\underset{\underline{\underline{\varepsilon}}}{\mathrm{p}} \otimes \underline{\underline{\varepsilon}}^{\mathrm{p}}
$$

\section{IDENTIFICATION OF MATERIAL PARAMETERS}

\section{Identification of the parameters of the kinetic law of strain induced martensitic} transformation

The parameters have been identified from the curve of martensite content versus the inelastic strain. The inelastic strain is decomposed into plastic strain $\varepsilon^{\mathrm{p}}$ and the phase transformation strain $\varepsilon^{\mathrm{PT}}$ according to the relationship:

$$
\underline{\varepsilon}^{i n e l}=\underline{\varepsilon}^{p}+\xi_{\underline{\varepsilon}}^{\mathrm{PT}}
$$

The martensite content can be plotted as a function of plastic strain. Linearization of the region II of the sigmoidal curve is carried out by using the least square method. The slope 


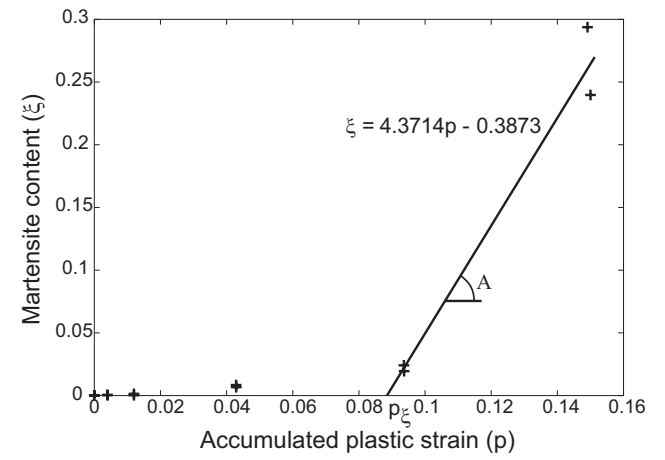

FIGURE 3: Identification of parameters of the kinetic law of martensitic transformation at $77 \mathrm{~K}$ for $316 \mathrm{~L}$

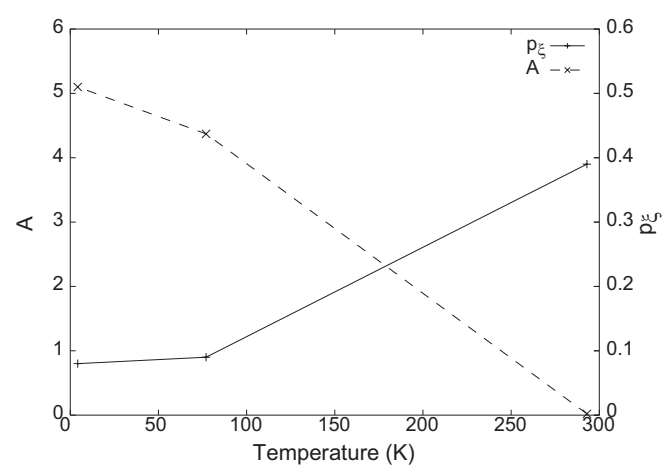

FIGURE 4: Intensity parameter (A) and plastic strain threshold $\left(\mathrm{p}_{\xi}\right)$ as a function of temperature

of the line and the intersection with the $\mathrm{x}$-axis correspond to the function $\mathrm{A}$ and threshold $\mathrm{p}_{\xi}$, respectively. As an example, the identification of the parameters for grade $316 \mathrm{~L}$ stainless steel at $77 \mathrm{~K}$ is shown in Fig. 3. The identification has been carried out for 3 temperatures (RT, $77 \mathrm{~K}, 4.2 \mathrm{~K}$ ). The evolution of two parameters describing the plastic strain induced martensitic transformation, $\mathrm{p}_{\xi}$ and $\mathrm{A}$ is given in Fig. 4.

\section{Identification of the material parameters of the damage evolution law}

The identification of the parameters of the kinetic law of damage evolution is based on the loading/unloading uniaxial tensile tests [9]. As an example, tensile curve obtained at $4.2 \mathrm{~K}$ from a $316 \mathrm{~L} 0.25 \mathrm{~mm}$ thick fine gauge sheet is shown in Fig. 5. The corresponding evolution of the damage parameter $\mathrm{D}$ as a function of the accumulated plastic strain is illustrated in Fig. 6. Again, the identification has been carried out for 3 temperatures $(293 \mathrm{~K}, 77 \mathrm{~K}$ and $4.2 \mathrm{~K})$. The approximate evolution of $\mathrm{S}$ with temperature is shown in Fig. 7.

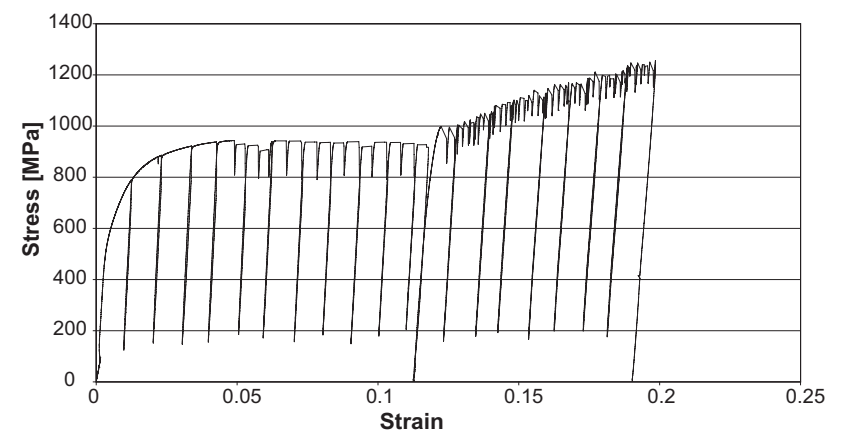

FIGURE 5: A tensile curve obtained at $4.2 \mathrm{~K}$ for $316 \mathrm{~L}$ fine gauge sample.

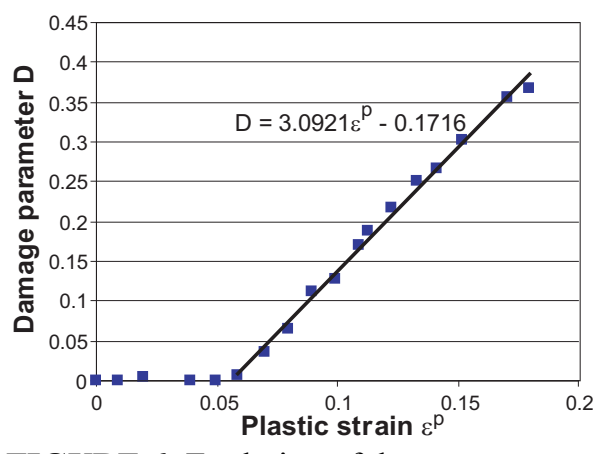

FIGURE 6: Evolution of damage parameter $\mathrm{D}$ as a function of plastic strain at $4.2 \mathrm{~K}$

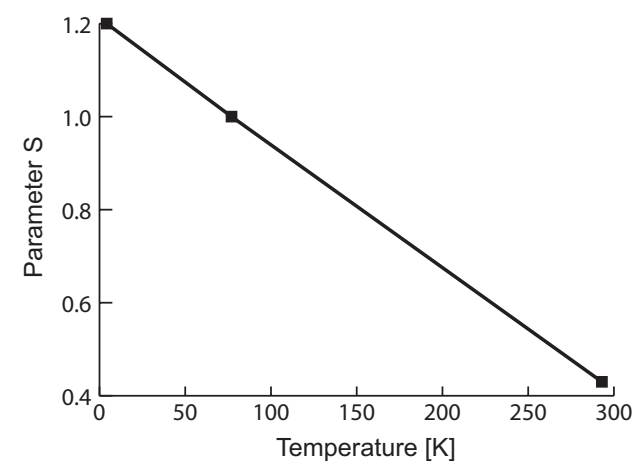

FIGURE 7: Evolution of the parameter $\mathrm{S}$ with temperature $\mathrm{T}$. 
The process of identification of the parameters $\mathrm{C}_{1}$ and $\mathrm{C}_{2}$ is done with the same method in 2 orthogonal directions. The method has been applied to 316 $\mathrm{L}$ stainless steel samples, extracted from a thin sheet in the longitudinal and in the transverse directions. The parameters $\mathrm{C}_{1}$ and $\mathrm{C}_{2}$, corresponding to two orthogonal directions, measured at three temperature levels are presented in Table 1.

TABLE 1 Parameters $C_{1}$ and $C_{2}$ as a function of temperature $T$

\begin{tabular}{|l|c|c|c|}
\hline Temperature [K] & 293 & 77 & 4.2 \\
\hline $\mathrm{C}_{1}$ (Longitudinal) & 1.51 & 1.0 & 0.90 \\
\hline $\mathrm{C}_{2}$ (Transverse) & 1.43 & - & 0.88 \\
\hline
\end{tabular}

\section{APPLICATION TO THIN-WALLED CRYOGENIC BELLOWS}

The constitutive model has been tested on thin-walled stainless steel corrugated shells, known as bellows expansion joints. They are used as compensation elements in the interconnections of the Large Hadron Collider at CERN. As an example, a half convolution of a typical U-type bellows has been considered (Fig. 8). The bellows was subjected to an axial displacement cycle between $-25 \%$ and $+62.5 \%$ of its initial length (denotes compression and + denotes extension).

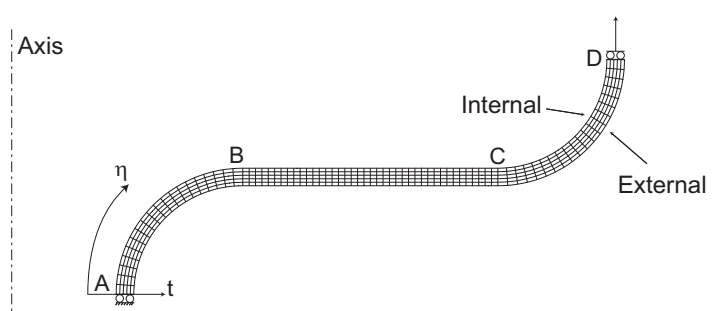

FIGURE 8. Half convolution of a bellows expansion joint (A-root, D-crest).

Since the $\mathrm{C} 1$ and $\mathrm{C} 2$ values identified at $4.2 \mathrm{~K}$ in both principal directions (longitudinal and transverse) are similar and the final values - after the hydro-forming process - are not yet known, the simulation of the orthotropic damage evolution gives nearly identical results as the isotropic model. To illustrate better the nature of the orthotropic model an arbitrary assumption has been made that the parameters $\mathrm{C} 1$ and $\mathrm{C} 2$ (after the hydroforming of bellows at room temperature) are equal at $4.2 \mathrm{~K}$ to 1.1 and 0.9 , respectively. The relevant profiles of damage evolution are shown in Figs. 9, 10.

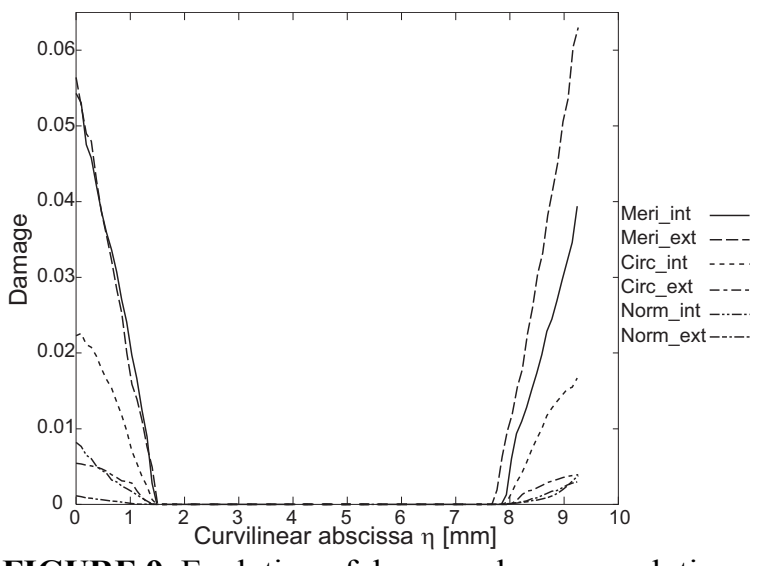

FIGURE 9. Evolution of damage along convolution (from point $\mathrm{A}$ to point $\mathrm{D}$ ) at $4.2 \mathrm{~K}$ (orthotropic case)

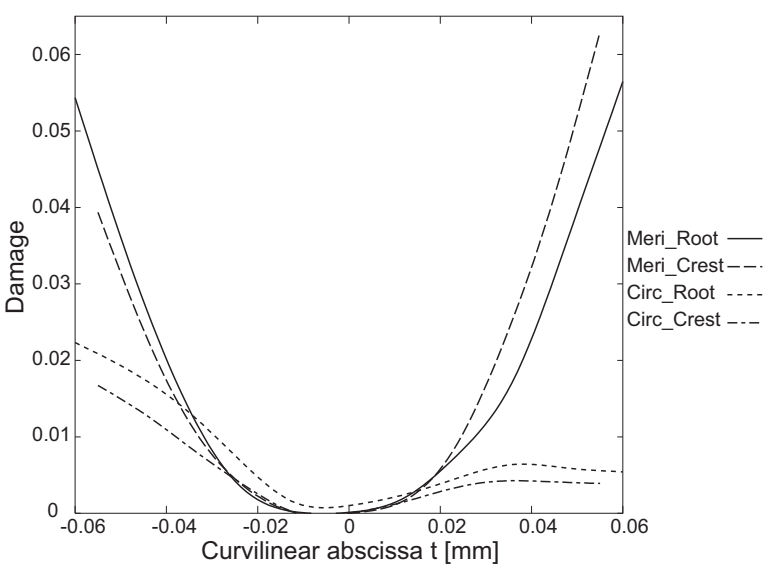

FIGURE 10. Evolution of damage through the thickness ( 0 corresponds to the mid-surface) at $4.2 \mathrm{~K}$ (orthotropic case) 


\section{CONCLUSIONS}

The constitutive modeling of two out of three phenomena occurring at cryogenic temperatures in ductile materials (stainless steels) was presented:

- plastic strain induced martensitic transformation $\left(\gamma \rightarrow \alpha^{\prime}\right)$,

- evolution of plastic strain induced orthotropic ductile damage.

In order to describe correctly the evolution of damage in thin-walled corrugated shells a second order damage tensor $\underline{\underline{D}}$ has been introduced. The relevant kinetic law of damage evolution for orthotropic damage and the associated potential of dissipation have been derived. The orthotropic law is a generalization of the well known isotropic law of damage evolution, introduced by Chaboche [8] and Lemaitre [9]. The damage rate is driven by the accumulated plastic strain rate. Thus, the principal directions of damage are decoupled with respect to the principal directions of plastic strains. Orthotropic damage develops in a two-phase material, that contains the ductile austenitic $\gamma$ matrix and the elastic $\alpha$ ' martensite inclusions. In the constitutive model presented in this paper, the damage is decoupled with respect to the martensitic transformation. It has been assumed that phase transformation does not influence the principal directions of damage. In order to reflect the initial texture of the material the Hill yield surface can be applied. Moreover, the effect of plastic strains on the shape of the yield surface (plasticity induced anisotropy) can be taken into account by more complex formulations [11-13]. Thus, a combination of the initial (texture induced) anisotropy and plasticity induced anisotropy will fully describe the process of plastic flow in thin-walled shell structures at cryogenic temperatures. In its present form the model is suitable mainly for the temperature range between $2 \mathrm{~K}$ and $77 \mathrm{~K}$ (in this range the kinetics of the phase transformation combined with the rate independent plasticity gives the correct results). The material parameters, represented by the tensor $\underline{\underline{\mathrm{C}}}$, that describes the anisotropy of damage, were identified for three temperature levels $(293 \mathrm{~K}, 77 \mathrm{~K}$ and $4.2 \mathrm{~K}$ ). The model has been extensively used to predict behavior and damage evolution in the bellows expansion joints designed for the interconnections of the Large Hadron Collider.

\section{REFERENCE}

1. Reed, R. P. and Walsh, R. P., Tensile strain rate effect in liquid helium, Adv. Cryogenic Engineering Materials, 34, p. 199-208 (1988).

2. Reed, R. P. and Simon, N. J., Discontinuous yielding in austenitic steels at low temperatures, International Cryogenic Material conference, Boulder, 2, p. 851-863 (1988).

3. Olson, G. B. and Cohen, M., Kinetics of strain-induced martensitic nucleation, Metallurgical transactions, 6A, p. 791-795 (1975).

4. Hill, R., The mathematical theory of plasticity, Clarendon Press, Oxford, 1950.

5. Hill, R., Theoretical plasticity of textured aggregates, Math. Proc. Camb. Phil. Soc., 85, p. 175191, (1979)

6. Garion, C., Skoczen, B., Modeling of plastic strain induced martensitic transformation for cryogenic applications, Jour. Applied Mechanics, 69 (2002).

7. Garion, C., Skoczen, B., Orthotropic damage and phase transformation in ductile materials at cryogenic temperatures, Proceedings of the ABDM, Cracow, Poland, 2002.

8. Chaboche, J. L., Continuum damage mechanics, Jour. Applied Mechanics, 55, p. 59-64 (1988).

9. Lemaitre, J., A course on damage mechanics, Springer-Verlag, Berlin and New York, 1992.

10. Murakami, S., A continuum mechanics theory of anisotropic damage, Yielding, damage, and failure of anisotropic solids, Proceedings of the IUTAM/ICM symposium, 1990, p. 465-482.

11. Edelman, F. and Drucker, D. C., Some extensions to elementary plasticity theory, J. Frankl. Inst., 251, p. 581-605 (1951).

12. Baltov, A. and Sawczuck, A., A rule of anisotropic hardening, Acta. Mech., 1, p. 81-92 (1965).

13. Mroz, Z. and Niemunis, A., On the Description of Deformation Anisotropy of Materials, Yielding, Damage and failure of anisotropic solids, p. 171-186 (1990). 